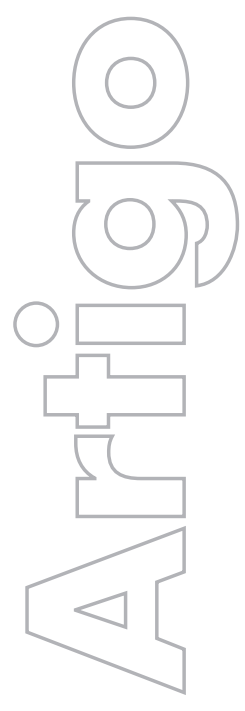

Modelos em geografia física: conceitos e aplicações na previsão de escorregamentos

\author{
Bianca Carvalho Vieira \\ USP \\ Tiago Damas Martins \\ UFPR
}

revista

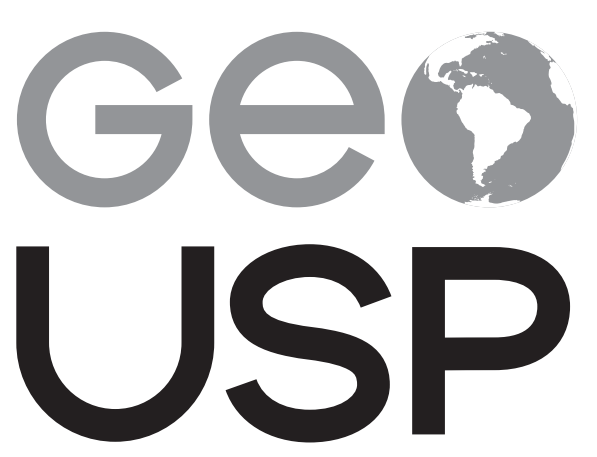

espaço e tempo

Volume $20 \cdot n^{\circ} 1(2016)$
Como citar:

VIEIRA, B. C.; MARTINS, T. D. Modelos em geografia física: conceitos e aplicações na previsão de escorregamentos. Geousp - Espaço e Tempo (Online), v. 20, n. 1, p. 194206, mês. 2016. ISSN 2179-0892.

Disponível em: http://www.revistas.usp.br/geousp/article/ view/102616. DOI: http://dx.doi.org/10.11606/issn.21790892.geousp.2016.102616.

\section{(c) (i) (5)}

Este artigo está licenciado sob a Creative Commons Attribution 4.0 License. 


\title{
Modelos em geografia física: conceitos e aplicações na previsão de escorregamentos
}

\section{Resumo}

Os diferentes tipos de modelo têm relação direta com o grau de abstração da realidade, estruturados desde formas mais simples, concretas e específicas, que transformam apenas a escala da realidade até em modelos conceituais, pela aplicação de fórmulas matemáticas. Entre todos, aqueles de base física, que descrevem fisicamente os processos por meio de equações e desconsideram a subjetividade, têm sido adotados em estudos de movimento de massa, por exemplo. $\bigcirc$ objetivo deste trabalho é discutir brevemente a fundamentação dos conceitos de modelo usados em geografia física, destacando dois modelos aplicados a estudos sobre escorregamentos e com resultados positivos nos testes. Primeiramente, discutiu-se o modelo clássico de correlação entre a pluviosidade e a ocorrência de escorregamentos. Depois, o modelo matemático em base física Shalstab avalia a possibilidade de escorregamentos rasos. Por fim, exemplificam-se os resultados desses modelos aplicados no Brasil.

Palavras-chave: Modelos em geografia física. Análise quantitativa. Modelo de correlação de chuva. Modelos matemáticos. Shalstab.

\section{Models in physical geography: concepts and applications to predict landslides}

\begin{abstract}
The different types of models have a direct relationship with the degree of abstraction of reality, occurring from simple, concrete and specific ways in which reality is turned only in scale level, until the development of conceptual models by applying mathematical formulations. Among those, the physically based models describe the physical processes through equations disregard any subjectivity and have been adopted, for example, on studies of mass movement. Thus, this paper aims to discuss briefly the concepts of models used in Physical Geography, highlighting two models applied in studies related to landslides that have demonstrated positive results in their tests. First it is discussed the classical model of correlation between rainfall and the occurrence of landslides. The second
\end{abstract}


one, it is the physically based model Shalstab, which assesses the susceptibility of occurrence of shallow landslides. Finally, are presented examples of results of these models applied in Brazil.

Keywords: Models in Physical Geography. Quantitative Analysis. Rainfall Correlation Model. Mathematical Models. Shalstab.

\section{Introdução}

Durante as décadas de 1950 e 1960, a geografia passou pela chamada Revolução Quantitativa e Teorética, em que foram realizadas importantes transformações em seus objetivos e métodos científicos. Tais transformações, segundo Christofoletti (1985), ocorreram na tentativa de incluir a geografia no contexto científico global por meio de um maior rigor na aplicação da metodologia científica, do desenvolvimento de teorias, do uso de técnicas estatísticas e matemáticas, da abordagem sistêmica e do uso de modelos.

Como destaca Burton (1992), a principal consequência dessa Revolução foi a "matematização da disciplina", pois, mesmo que o desenvolvimento de teorias seja parte essencial do método científico, a quantificação oferece uma técnica pela qual a teoria pode ser desenvolvida e aperfeiçoada. Para alguns pesquisadores, com essa Revolução surge a dicotomia qualitativa-quantitativa separando, por exemplo, a análise racional da percepção intuitiva (Burton, 1992).

Por meio dessas transformações, a Revolução Quantitativa e Teorética permitiu uma visão mais clara de uma determinada feição complexa da realidade e o desenvolvimento e aperfeiçoamento de teorias anteriormente propostas, mas não comprovadas (Taylor, 1977). Um exemplo é o questionamento do "modelo" de ciclo de erosão, desenvolvido por Willian Morris Davis, que passou a ser testado por meio de diferentes técnicas e pelo desenvolvimento de outros modelos da evolução da paisagem que tentaram conhecer e explicar as variáveis envolvidas nesse sistema (Taylor, 1977). A exemplo da geografia, a análise quantitativa permite lidar com o um imenso volume de dados, contribuindo na "verificação das relações entre os fenômenos estudados e a sua distribuição no espaço" (Gerardi; Silva, 1981, p. 22).

Assim, os modelos são importantes instrumentos para estudar os sistemas complexos da geografia, pois permitem estruturá-los para compreender melhor suas variáveis e suas interligações (Gerardi; Silva, 1981; Christofoletti, 1985, entre outros). Entretanto, alguns problemas na geografia devem ser bem avaliados para que não ocorra um abuso no uso de modelos, havendo uma imprecisão na formação ou reformulação de teorias (Harvey, 1969). Dessa forma, este trabalho tem como principal objetivo fazer uma leitura sobre os modelos na geografia física, tendo como exemplo os processos geomorfológicos de movimentos de massa. Na primeira etapa, são descritos os principais conceitos e definições dos modelos, suas principais características e funções e as diferentes classes. Na segunda etapa, há dois diferentes tipos de modelos aplicados a um mesmo processo, nesse caso, os escorregamentos. Tal processo, aqui selecionado, justifica-se pela sua importância em diferentes ramos da geografia (geomorfologia, climatologia, pedologia, entre outras) que procuram compreender a atuação dos seus agentes controladores (por exemplo, geologia e propriedades dos solos), explicar o seu papel na evolução da paisagem, e prever sua ocorrência e magnitude que causam frequentemente danos sociais e econômicos. 


\section{Modelos: conceitos e definições}

Para Minshull (1975), existe um problema na definição de modelos em função das diferentes abordagens científicas e da ausência de qualquer avaliação comparativa entre elas. Consequentemente, os modelos aparecem na literatura com diferentes definições, tais como: uma hipótese, uma lei, uma teoria formalizada, uma descrição de um fenômeno em termos matemáticos, uma representação, uma abstração, uma equação, um caminho de olhar as coisas, uma ideia estruturada, uma analogia e uma versão simplificada da realidade (Minshull, 1975).

Modelo pode ser definido como uma estrutura simplificada do funcionamento de um aspecto do mundo real (Haggett; Chorley, 1967; Harvey, 1969; Thomas; Huggett, 1980; entre outros), sendo uma aproximação reduzida do fenômeno, uma vez que, não incluiu todas as observações ou medidas associadas, obscurece detalhes acidentais e destaca somente os aspectos fundamentais da realidade (Haggett; Chorley, 1967).

A definição de modelo pode também contemplar a sua relação com a teoria, uma vez que é uma formalização e/ou uma estruturação simplificada de uma teoria que pode, por sua vez, ser representada por um ou mais modelos, permitindo a manipulação de deduções complexas (Harvey, 1969). Quanto maior o número de variáveis menos funcional é o modelo. Entretanto, é importante destacar que, devido à impossibilidade de considerar todos os detalhes do sistema, muitas vezes o modelo é inicialmente construído de forma simples para depois incorporar informações que o tornam mais complexo (Perin Filho, 1995).

Existem modelos e teorias com estruturas, propriedades e fenômenos idênticos, e outros com propriedades diferentes das do objeto real (Harvey, 1969). Para o autor, o modelo pode refletir a teoria parcialmente, sendo considerado um experimento procedente de sua abstração, ou totalmente, servindo para transferi-la a um domínio mais comum, conhecido e controlável.

Segundo Christofoletti (1999), na elaboração de um esquema representativo (modelo), o que está sendo "representado" não é a realidade em si, mas sim a visão do "modelador", ou seja, como ele observa um determinado processo e quais as variáveis do sistema que são conhecidas e consideradas por ele para a estruturação do modelo.

\section{Modelos: características e funções}

Segundo Haggett e Chorley (1967) e Christofoletti (1999), as principais características dos modelos são: (a) seletividade, que consiste em selecionar as informações, descartando os aspectos menos importantes e considerando só os mais significativos e fundamentais do mundo real; (b) estruturação, em que os aspectos selecionados da realidade são tratados nos termos de suas conexões; (c) reaplicação, demonstra que o modelo não descreve apenas um caso, mas pode representar outros da mesma categoria (Haggett; Chorley, 1967; Christofoletti, 1999); (d) sugestivo, podem sugerir que as implicações do conjunto são maiores do que as possivelmente supostas por suas partes individuais e que há um potencial enunciativo para previsões sobre aspectos no mundo real; (e) simplicidade, devem ser fáceis de manipular e compreender, mas sem prejudicar a complexidade necessária para representar o sistema estudado; (f) analógicos, diferem do mundo real, mas mostram-no aproximadamente, para melhor compreendê-lo (Fernandes, 1996).

Como afirmam Moore, O'Loughlin e Burch (1988), a funcionalidade de um modelo está relacionada com o número de variáveis utilizadas. Quando há uma diminuição no número de variáveis de um determinado modelo, o seu desempenho, no que se refere aos resultados, apresenta uma acentuada melhora (Figura 1 e Quadro 1). 
Figura 1 - Relação hipotética entre a funcionalidade e o número de variáveis do modelo

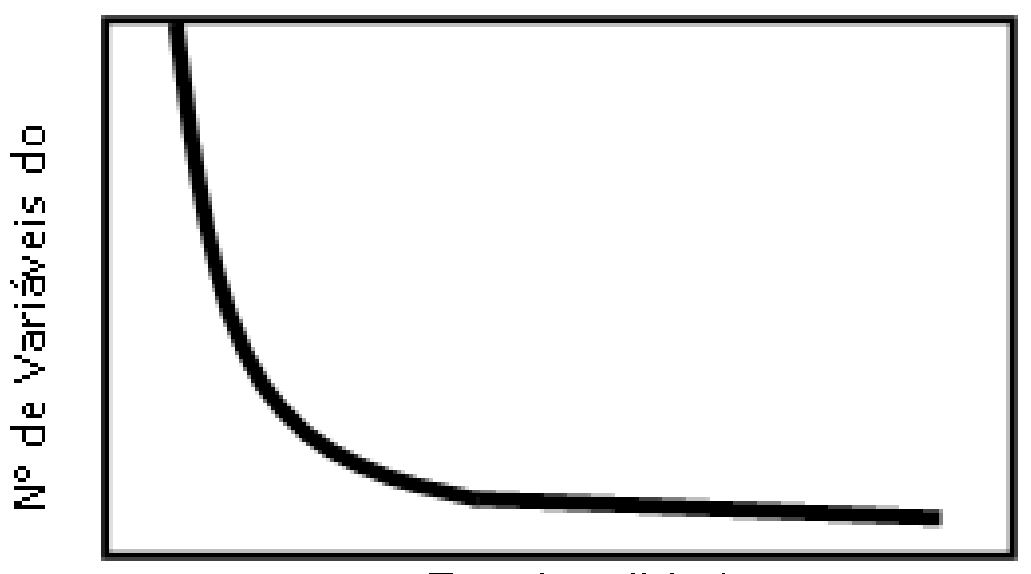

Funcionalidade

fonte: Adaptada de Moore, O'Loughlin e Burch (1988).

\section{Quadro 1 - Principais funções dos modelos}

\begin{tabular}{|c|c|}
\hline função & descrição \\
\hline psicológica & $\begin{array}{l}\text { permite ver e compreender determinado grupo de fenômenos, o que } \\
\text { não seria possível de outra forma, devido a sua magnitude e complexi- } \\
\text { dade }\end{array}$ \\
\hline aquisitiva & permite definir, coletar e ordenar as informações dentro do modelo \\
\hline $\begin{array}{l}\text { organizacional/ } \\
\text { fertilidade }\end{array}$ & permite extrair dos dados o máximo de informações \\
\hline lógica & ajuda a explicar determinado fenômeno \\
\hline normativa & compara algum fenômeno com outro, mais familiar \\
\hline sistemática & considera a realidade em termos de sistemas interligados \\
\hline construtiva & os modelos formam degraus para construir teorias e leis \\
\hline parentesco & promove a comunicação das ideias científicas \\
\hline comunicação & $\begin{array}{l}\text { proporciona um arcabouço em que as informações podem ser definidas, } \\
\text { ordenadas e relacionadas }\end{array}$ \\
\hline promissão & $\begin{array}{l}\text { gera novos enunciados e percepção de relações, tornando-se promissor } \\
\text { para se extrair dos dados o máximo de informações }\end{array}$ \\
\hline logicidade & ajuda a explicar como acontece e se encadeia determinado fenômeno \\
\hline adequação & permite adequação à análise pretendida \\
\hline previsibilidade & $\begin{array}{l}\text { muitos modelos permitem fazer previsões específicas como base para } \\
\text { decisões imediatas - importante função para planejamento }\end{array}$ \\
\hline
\end{tabular}

fonte: Haggett e Chorley (1967) e Christofoletti (1999). 


\section{Modelos: tipos e classes na geografia}

Os diferentes tipos de modelos têm uma relação direta com o grau de abstração da realidade, ocorrendo desde formas mais simples, concretas e específicas, em que a realidade é transformada apenas em nível de escala até a elaboração de modelos conceituais pela aplicação de modelos matemáticos, sendo esses últimos mais abstratos e gerais (Taylor, 1977; Thomas; Huggett, 1980).

Referente a esta abstração, Haggett e Chorley (1967) afirmam que o "valor dos modelos está diretamente relacionado ao seu nível de abstração e todos os modelos têm a necessidade constante de aperfeiçoamento". Esses autores dividem os modelos em descritivos e normativos. Os modelos descritivos podem ser estáticos, concentrados nas características do equilibrio estrutural, ou dinâmicos, concentrados nos processos e funções através do tempo (Haggett; Chorley, 1967). Os modelos (sistemas) na geografia, especificamente na geomorfologia, segundo Chorley (1967), podem ser classificados em naturais (ou análogos), fisicos e gerais.

Os sistemas (modelos) naturais análogos traduzem aspectos importantes de um determinado fenômeno em um sistema análogo natural (Chorley, 1967). Já os sistemas (modelos) físicos se baseiam na "perspectiva de que a pesquisa pode ser mais bem realizada pela dissecação da estrutura do problema geomórfico em suas partes supostamente componentes, de modo que o funcionamento de cada parte e as interações entre elas possam ser examinadas convenientemente, levando-se a uma síntese completa dos componentes num todo funcional" (Chorley, 1967; Christofoletti, 1999). Entre as subclasses desse grupo destacam-se os modelos de projetos experimentais e modelos matemáticos (determinísticos e estocásticos). Esses últimos representam a natureza por meio de equações matemáticas podendo ser divididos em determinísticos e estocásticos. Segundo Perin Filho (1995), a distinção entre modelos determinísticos e estocásticos é muito influenciada pela qualidade da informação, pelo controle que se tem sobre o sistema e pelo objetivo que se pretende alcançar com o estudo.

Segundo Chorley (1967), nos modelos matemáticos determinísticos o comportamento do sistema geográfico é controlado por leis físicas naturais e o resultado é previsto de forma exata, porquanto, "são baseados nas noções matemáticas clássicas de relações exatamente previsíveis, entre variáveis independentes e dependentes", e podem ser aferidos a partir de argumentações matematicamente lógicas (Chorley, 1967, p. 60).

Nos modelos matemáticos estocásticos, o valor numérico obtido para uma variável está associado a uma probabilidade de ocorrência. Assim, para cada entrada (input), existirão várias saídas (output), refletindo as incertezas do sistema, e os elementos podem estar associados a seus inputs, parâmetros, distribuições espaciais ou aos processos modelados (Christofoletti, 1999).

Os sistemas (modelos) gerais fornecem um quadro global do sistema, estabelecendo 
o grau de conhecimento sobre as partes componentes, interações entre os elementos e o funcionamento interativo entre as entradas e saídas (Christofoletti, 1999). São subdivididos em modelos caixa-preta, caixa-cinza e caixa-branca. Os modelos caixa-preta, segundo Chorley (1967), exigem pouca ou nenhuma informação detalhada dos componentes do sistema havendo somente o interesse na natureza das saídas resultantes das diferentes entradas, e sendo os resultados extraídos sem o conhecimento da estrutura interna do sistema. Um exemplo desse tipo de modelo é a regressão múltipla, que apresenta uma aplicação bastante rápida e associada a outras técnicas levam a combinação das variáveis envolvidas. Porém, sua desvantagem é o pequeno acréscimo sobre a compreensão do funcionamento do sistema, pois seus parâmetros não possuem interpretação física direta (Christofoletti, 1999).

Como exemplos clássicos, Chorley (1967) cita os modelos de equilíbrio dinâmico de G. K. Gilbert e o ciclo de Willian M. Davis. Ambos se baseiam em pressuposições intuitivas no tocante aos padrões comportamentais significantes dos conjuntos das formas de relevo sem uma investigação detalhada da natureza dos processos envolvidos (Chorley, 1967).

Os modelos caixa-cinza tentam tirar resultados sem o completo conhecimento do trabalho interno do sistema, mas a obtenção de informações específicas sobre as inter-relações entre os subsistemas permite identificar o comportamento do sistema sob diferentes condições de entrada (Chorley, 1967).

Finalmente, nos modelos caixa-branca a estrutura do sistema é construída pelo conhecimento das variáveis envolvidas e das suas relações. A rotina para estabelecer esses sistemas sintéticos começa com a identificação de certos elementos. Posteriormente, é feito o mapeamento das variáveis correlacionadas em conjuntos planos, e as direções de causalidade são indicadas mostrando a existência de relações entre os membros de um sistema e o ambiente vizinho (Chorley, 1967; Kirkby et al.,1987).

Os modelos baseados em processos são um tipo de modelo sintético, que descrevem os mecanismos de operações particulares que ocorrem no mundo. A partir do conhecimento dos processos, pode-se direcionar a escolha das variáveis, mostrando, por exemplo, que as relações internas do sistema são bem diferentes daquelas dadas pelo simples modelo de regressão. Esse tipo de modelo é construído a partir de um fluxo diagrama que representa estocagens físicas e/ ou fluxo de energia ou de material do mundo real (Kirkby et al., 1987).

\section{Uso de modelos preditivos para escorregamentos}

Em relação aos escorregamentos, dois casos são apresentados, sendo um que combina uma relação entre o volume de precipitação e a ocorrência de escorregamentos, e outro determinístico, apoiado em equações matemáticas. 


\section{Correlação precipitação pluvial e escorregamentos}

Um dos trabalhos clássicos na compreensão de escorregamentos foi realizado por Guidicini e Iwasa (1976) com base em modelos de correlação entre a pluviosidade e a ocorrência de escorregamentos em nove áreas: Serra de Caraguatatuba, Baixada Santista, rodovia dos Imigrantes, rodovia Anchieta, Serra de Maranguape, Região Metropolitana do Rio de Janeiro, Serra das Araras, sul de Minas Gerais e Vale do Tubarão.

Primeiramente, se identificaram os níveis de precipitação pluvial associados, ou não, aos escorregamentos. Segundo os autores, a precipitações com alta de intensidade (>250 mm e $300 \mathrm{~mm}$ ) são capazes de desencadear escorregamentos em qualquer circunstância. Essa correlação não é válida para os episódios de precipitação de menor intensidade, pois há uma intercalação de registros desses processos (Guidicini; Iwasa, 1976). Para correlacionar a pluviosidade com os escorregamentos foram introduzidos os dados históricos de precipitação, e realizadas análises baseadas no ciclo parcial e no ciclo total de chuva do ano em questão.

$\mathrm{Na}$ análise do ciclo parcial de precipitação foi considerado um somatório contínuo de valores, ou seja, os índices pluviométricos dos episódios e aqueles imediatamente anteriores ( 7 dias). $O$ resultado foi convertido em termos de percentagem da pluviosidade média anual em cada área, permitindo a comparação entre as nove áreas estudadas.

Por meio dessa análise, os autores concluem que os escorregamentos tendem a ocorrer nos índices de pluviosidade que se situam, em média, entre $8 \%$ e 17\% da pluviosidade média anual, e os demais episódios que não provocaram escorregamentos, entre $8 \%$ e $12 \%$ da pluviosidade média anual. Foi observado também, em algumas regiões, que os escorregamentos de grande magnitude (efeito catastrófico) estão associados a índices de precipitação de $23 \%$ acima da média anual. Já em outras áreas, os processos desta magnitude não foram deflagrados por índices acima da média.

$\mathrm{Na}$ análise do ciclo completo de precipitação, foram aplicados dois coeficientes que representam as porcentagens de precipitação pluvial em relação à média anual: (a) o coeficiente do ciclo $(\mathrm{Cc})$, razão entre o registro pluviométrico acumulado até a data do episódio e a média anual de pluviosidade, e (b) o coeficiente do episódio $(\mathrm{Ce})$ que representa a razão entre o registro pluviométrico do episódio e a média anual de pluviosidade.

Foram feitas também correlações entre todas as nove áreas estudadas com base no coeficiente do ciclo ( $\mathrm{Cc}$ ) e no coeficiente final (Cf). Os autores reuniram todos os dados disponíveis num só gráfico, plotando 101 pontos correspondentes aos episódios de chuva, dos quais 41 estão associados à ocorrência de movimentos de massa. Posteriormente foram definidas quatro faixas, A, B, C e D, que separam zonas com diferentes concentrações de episódios de escorregamentos, 100\%, $50 \%, 33 \%$ e $0 \%$ (Figura 2). 
Figura 2 - Correlação entre os episódios pluviométricos, o coeficiente final $(\mathrm{C} f)$ e as ocorrências de escorregamentos. Com base nessa análise, foi possível determinar quatro faixas ( $A, B, C$ e D) com diferentes concentrações de escorregamentos

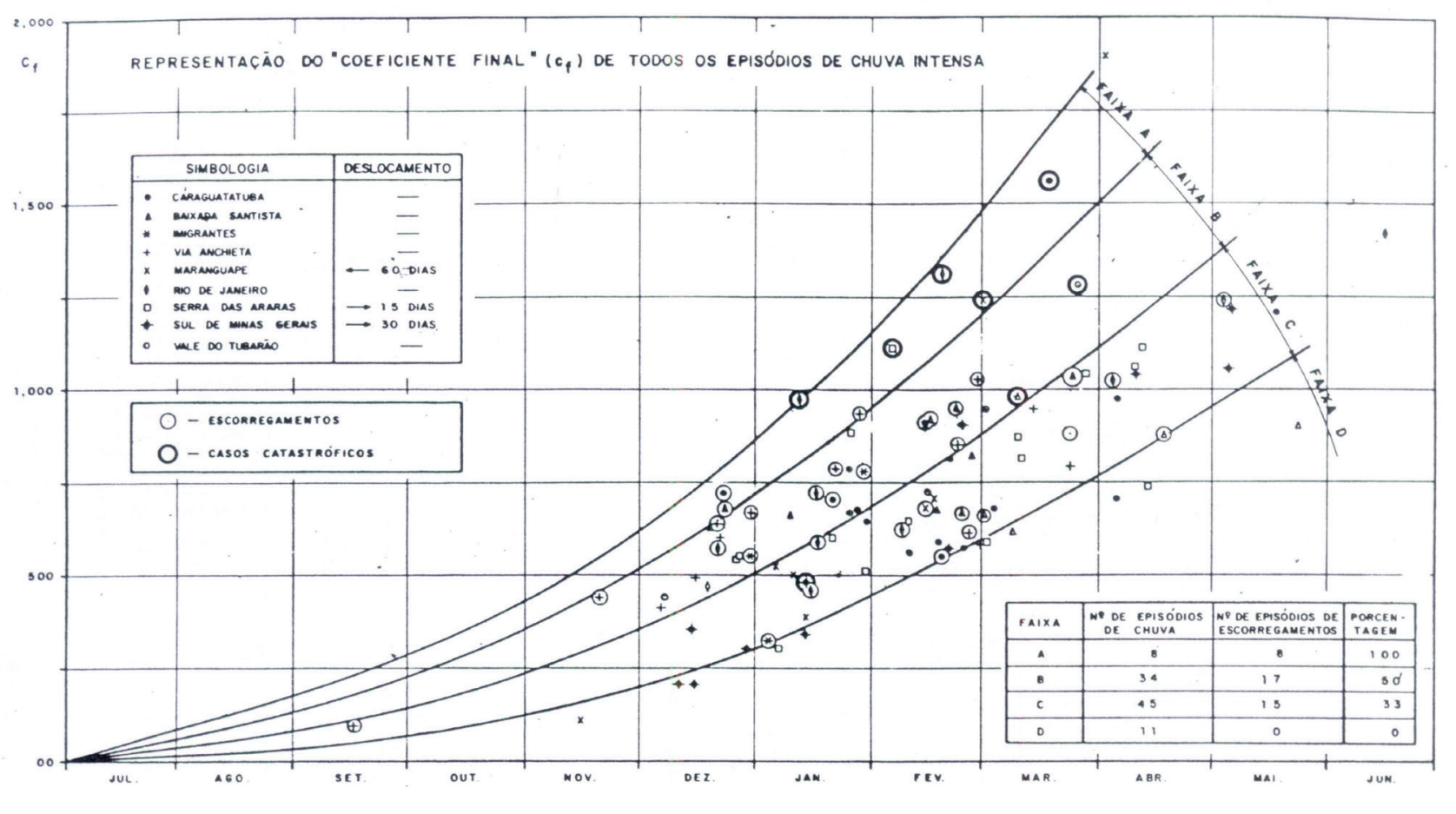

fonte: Guidicini e Iwasa (1976).

Com base na aplicação desses modelos de correlação, os autores puderam indicar algumas hipóteses sobre o papel da chuva na deflagração dos escorregamentos em encostas. Segundo eles, o histórico de pluviosidade desempenha um papel importante no desencadeamento dos escorregamentos, pois a umidade antecedente no solo interfere diretamente no avanço da frente de saturação, e esta, por sua vez, poderá reduzir a coesão dos solos levando a ruptura da encosta (Guidicini; Iwasa, 1976).

Esse modelo tem como principais vantagens: (a) a rapidez na aplicação e na obtenção dos dados; (b) a simplicidade quanto à estrutura que facilita o seu uso por diferentes usuários como, por exemplo, órgãos públicos; (c) a identificação de níveis de chuvas diretamente relacionados à deflagração de escorregamentos, e (d) o levantamento de hipóteses sobre o papel da chuva e da umidade antecedente do solo na deflagração dos escorregamentos. Essas hipóteses só foram diagnosticadas devido à correlação entre os escorregamentos e as chuvas anteriores à ruptura.

Quanto as suas limitações, por considerar somente um único agente deflagrador dos escorregamentos, nesse caso a chuva, o modelo se torna muito simples. Dessa forma, alguns dos resultados obtidos não foram capazes de explicar a distribuição e a mag- 
nitude dos escorregamentos, uma vez que o modelo não incluiu, por exemplo, as propriedades dos solos. Uma segunda limitação é quanto a sua aplicação em outras áreas com registros pluviométricos diferentes, pois existe a dificuldade desse modelo em ser utilizado sob condições fora da faixa dos dados originais.

Uma terceira importante limitação refere-se ao pequeno acréscimo à compreensão do funcionamento do sistema e consequentemente à reformulação e/ou estruturação de novas teorias. Por último, pode-se mencionar também, a exigência da coleta de séries longas de chuvas e do mapeamento dos escorregamentos para a aplicação do modelo de correlação e elaboração das cartas de periculosidade em áreas com ausência desses dados.

\section{Modelo em base física}

Um dos métodos que vem sendo utilizado para previsão de áreas suscetíveis a escorregamentos rasos são os modelos matemáticos em bases físicas que descrevem fisicamente os processos por meio de equações matemáticas, desconsiderando uma possível opinião subjetiva e direta do pesquisador. Tais modelos preveem a suscetibilidade sob diferentes cenários de uso e eventos climáticos, por exemplo, independente da ocorrência anterior dos processos (Guzzetti et al., 1999; Van Westen, 2004).

Entre os modelos, o Shalstab (Shallow Landsliding Stability Model), desenvolvido na década de 1990 a partir dos trabalhos de Dietrich et al. (1993, 1995), Montgomery e Dietrich (1994) e Dietrich e Montgomery (1998), é um dos modelos mais aplicados mundialmente, e no Brasil foi utilizado primeiramente por Guimarães et al. (2003) e, depois, por Vieira (2007), Guimarães et al. (2009), Zaidan e Fernandes (2009), Listo e Vieira (2012), Michel, Kobiyama e Goerl (2014), Vieira e Ramos (2015), entre outros

$\bigcirc$ Shalstab representa espacialmente a suscetibilidade de ocorrência de escorregamentos translacionais rasos, em escala de bacia hidrográfica, a partir da combinação de um modelo de estabilidade baseado na Equação do Talude Infinito, e de um modelo hidrológico em condição estável (steady-state) (equação 1).

$$
\log \frac{\mathrm{Q}}{\mathrm{T}}=\left(\log \frac{\operatorname{sen} \theta}{\mathrm{a} / \mathrm{b}}\left(\frac{\mathrm{C}^{\prime}}{\rho_{\mathrm{w}} \mathrm{gz} \cos ^{2} \theta \tan \phi}+\frac{\rho_{\mathrm{s}}}{\rho_{\mathrm{w}}}\left(1-\frac{\tan \theta}{\tan \phi}\right)\right)\right) \quad \text { (equação 1) }
$$

Na qual, Q é chuva crítica necessária para a ruptura [mm]; T é a transmissividade do solo [ $\left.\mathrm{m}^{2} / \mathrm{dia}\right]$; $a$ é a área de contribuição [ $\left.\mathrm{m}^{2}\right]$; $b$ é o comprimento do contorno da célula [m]; $c$ é a coesão do solo [Pa]; $\theta$ é o ângulo da encosta $\left[{ }^{\circ}\right] ; \rho_{w}$ é a densidade da água $\left[\mathrm{kN} / \mathrm{m}^{3}\right] ; \boldsymbol{\rho}_{s}$ densidade do solo $\left[\mathrm{kN} / \mathrm{m}^{3}\right]$; $g$ é a gravidade $\left[\mathrm{m} / \mathrm{s}^{2}\right]$; $z$ é a espessura do solo e $\phi$ é o ângulo de atrito interno $\left[^{\circ}\right]$.

Na aplicação desse modelo, tem-se considerado diferentes cenários variando valores dos parâmetros físicos do solo (Figura 2), por exemplo, e correlacionando os diferentes cenários com a ocorrência de cicatrizes de escorregamentos, ou seja, como se baseia apenas 
em equações matemáticas, para sua aplicação não é necessária a inclusão da ocorrência de escorregamentos pretéritos dentro da sua análise, como necessário em outros modelos (por exemplo, ferramentas estatísticas).

Esse modelo, do tipo determinístico, apresenta como principais potencialidades, (a) a versatilidade; (b) a sua estruturação que permite modificações; (c) a rapidez na obtenção de resultados e precisão na localização dos escorregamentos rasos; (d) a inclusão de diversos fatores que teoricamente influenciam na estabilidade das encostas, tais como: coesão dos solos, fluxos de água na encosta e forma da encosta; (e) a análise de sensitividade do modelo, que permite avaliar quais os fatores mais significativos para ruptura.

Entre as principais limitações, destacam-se: (a) a qualidade dos dados para alimentação do modelo; (b) a discretização de processos contínuos (por exemplo, fluxo de água em encostas); (c) dificuldade de representação matemática de alguns aspetos e processos físicos (por exemplo, estruturas geológicas) e (d) as aproximações necessárias para o seu desenvolvimento e aplicação (por exemplo, considerar somente o fluxo subsuperficial paralelo à encosta).

Figura 2 - Exemplo de aplicação do modelo Shalstab numa bacia hidrográfica, com a indicação de áreas potencialmente instáveis, variando em cada cenário (A1 a B3) as propriedades físicas do solo e resultando em dimensões diferentes as classes de instabilidade
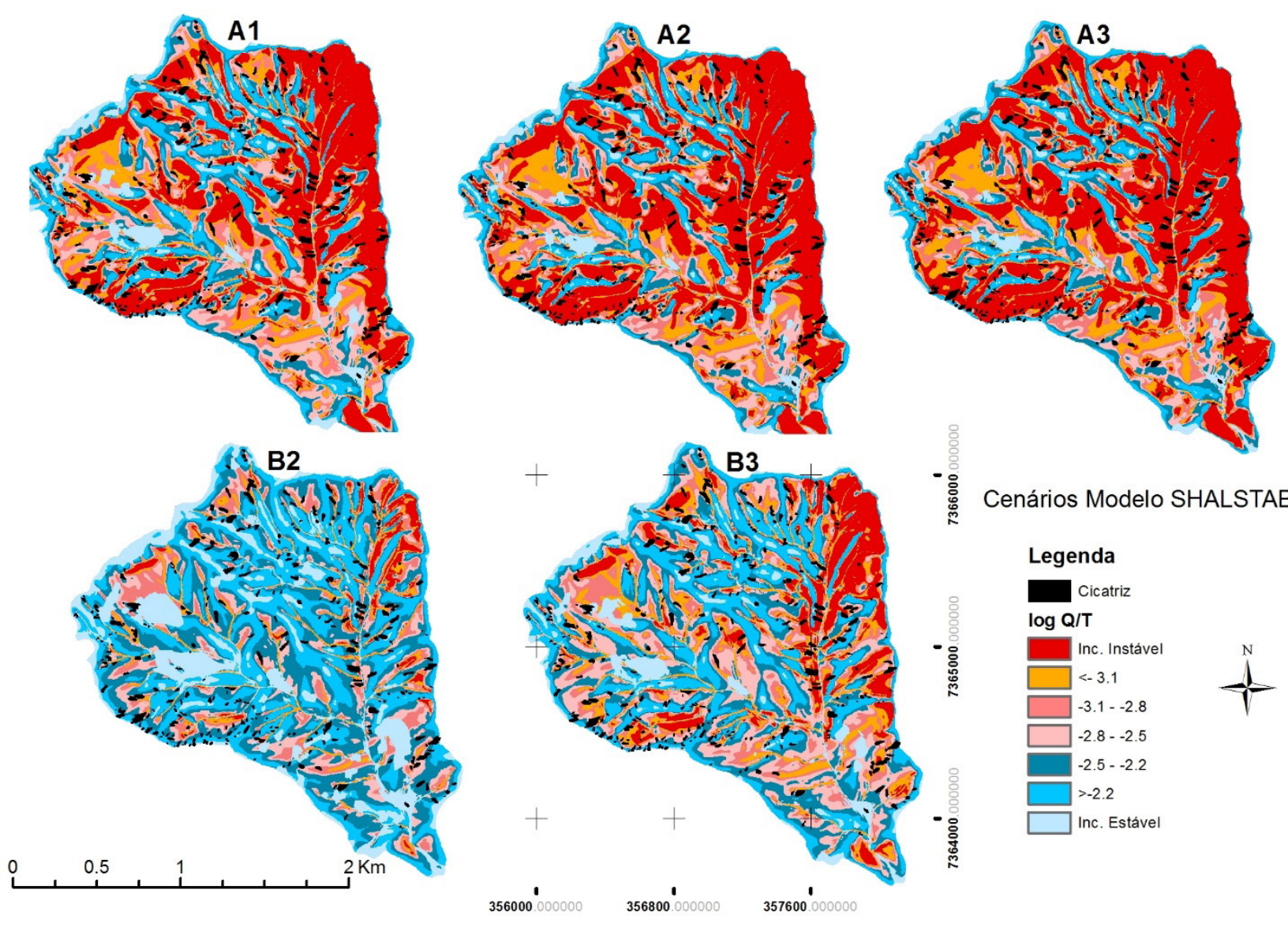

Cenários Modelo SHALSTAB

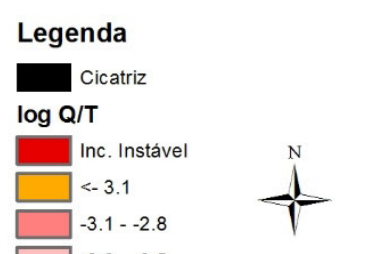

fonte: Vieira (2007). 


\section{Considerações finais}

Os modelos são instrumentos importantes para entender os fenômenos naturais e sociais, pois são capazes de representar simplificadamente a realidade. Essa representação pode ser feita por aproximação a alguns aspectos do mundo real, seleção de uma gama de variáveis e eliminação de detalhes incorporados à complexidade dos fenômenos naturais.

Todos os tipos de modelos apresentados neste trabalho têm potencialidades e limitações de acordo com o nível de abstração envolvido. As próprias características e funções dos modelos demonstram as vantagens e as desvantagens de seu uso. Por exemplo, ao mesmo tempo em que a simplicidade do modelo permite manipular e compreender o mundo real e complexo, elimina fatores que podem ser considerados de extrema importância no sistema modelado.

De uma maneira geral, os modelos concorrem para o desenvolvimento de conceitos; formulação e/ou reformulação de teorias; identificação de hipóteses sobre um determinado processo natural; análise da importância dos diversos parâmetros envolvidos no problema e maior percepção do papel das variáveis do sistema, assim como de suas conexões.

Ambos os modelos aqui exemplificados têm diferentes níveis de abstração e generalização que os tornam mais simples ou mais complexos, exigem maior ou menor quantidade e qualidade de dados e, portanto, mais ou menos eficientes na previsão de escorregamentos em encostas.

\section{Referências}

BURTON, I. The quantitative revolution and theoretical geography. In: DAVIES, W. K. D. (Ed.). The conceptual revolution in Geography. Londres: University of London Press, 1992. p. 140-156.

CHORLEY, R. J. Models in Geomorphology. In: CHORLEY, R. J.; HAGGETT, P. (Ed.). Models in Geography. Londres: Methuen \& Co., 1967. p. 43-96.

CHRISTOFOLETTI, A. Modelagem de sistemas ambientais. São Paulo: Edgard Blucher, 1999.

As perspectivas dos estudos geográficos. In: CHRISTOFOLETTI, A. (Ed.). Perspectivas da Geografia. São Paulo: Difel, 1985. p. 11-36.

DIETRICH, W. E.; MONTGOMERY, D. R. Shalstab: a digital terrain model for mapping shallow landslide potential. Cary, NC: NCASI, 1998. Technical Report.

. et al. A Process-Based Model for Colluvial Soil Depth and Shallow Landsliding Using Digital Elevation Data. Hydrological Processes, v. 9, p. 383-400, 1995.

. et al. Analysis of erosion thresholds, channel networks and landscape morphology using a digital terrain model. Journal of Geology, Chicago, v. 101, n. 2, p. 259-278, mar. 1993.

FERNANDES, N. F. Modelagem Matemática em geomorfologia: potencialidades e limitações. Sociedade E Natureza, Uberlândia, MG, v. 15, p. 222-227, 1996. 
GERARDI, L. H. O.; SILVA, B. C. N. Quantificação em geografia. São Paulo: Difel, 1981.

GUIDICINI, G.; IWASA, O. Y. Ensaio de correlação entre pluviosidade e escorregamentos em meio tropical úmido. São Paulo: IPT, 1976. (IPT. Publicação, 1080).

GUIMARÃES, R. F. et al. Análise temporal das áreas susceptíveis a escorregamentos rasos no Parque Nacional da Serra dos Órgãos (RJ) a partir de dados pluviométricos. Revista Brasileira de Geociências, v. 39, p. 192-200, 2009. et al. Parameterization of soil properties for a model of topographic controls on shallow landsliding: application to Rio de Janeiro. Engineering Geology, v. 69, p. 99-108, 2003.

GUZZETTI, F. et al. Landslides hazard evaluation: a review of current techniques and their application in a multi-scale study, Central Italy. Geomorphology, v. 31, n. 1-4, p. 181216, 1999. Disponível em: <doi: 10.1016/S0169-555X(99)00078-1>. Acesso em: 12 fev. 2016.

HAGGETT, P.; CHORLEY, R. J. Models, paradigms and the new Geography. In: CHORLEY, R. J.; HAGGETT, P. (Ed.). Models in Geography. Londres: Methuen e Co., 1967. p. 19-41.

HARVEY, D. Explanation in Geography. Londres: Edward Arnold, 1969.

KIRKBY, M. J. et al. Computer simulation in Physical Geography. Chichester: Wiley, 1987.

LISTO, F. L. R.; VIEIRA, B. C. Mapping of risk and susceptibility of shallow-landslide in the city of São Paulo, Brazil. Geomorphology, v. 169-170, p. 30-44, 2012. Disponível em: <doi: 10.1016/j.geomorph.2012.01.010>. Acesso em: 12 fev. 2016.

MICHEL, G. P.; KOBIYAMA, M.; GOERL, R. F. Comparative analysis of Shalstab and SINMAP for landslide susceptibility mapping in the Cunha River basin, southern Brazil, Journal of Soils and Sediments, v. 14, n. 7, p. 1266-1277, 2014. Disponível em: <doi: 10.1007/s11368-014-0886-4. 2014>. Acesso em: 12 fev. 2016.

MINSHULL, R. An introduction to models in Geography. New York: Longman Group Limited, 1975.

MONTGOMERY, D. R.; DIETRICH, W. E. A physically based model for the topographic control on shallow landsliding. Water Resources Research, v. 30, n. 4, p. 1153-1171, 1994.

MOORE, I. D.; O'LOUGHLIN, E. M.; BURCH, G. J. A Counter-Based Topographic Model for Hydrological and Ecological Applications. Earth Surface and Processes and Landforms, v. 13, p. 305-320, 1988.

PERIN FILHO, C. Introdução à simulação de sistemas. Campinas, SP: Editora Unicamp, 1995. (Coleção livro-texto).

TAYLOR, P. J. Quantitative methods in Geography: an introduction to spatial analysis. Boston: Houghton Mifflin Company, 1977.

THOMAS, R. W.; HUGGETT, R. J. Modelling in Geomorphology: a mathematical approach. London: Harper and Row, 1980. 
VAN WESTEN, C. J. Geo-information tools for landslide risk assessment: an overview of recent development. In: LACERDA, W. A. et al. (Ed.). Landslides: Evaluation and Stabilization. Londres: Taylor E Francis Group, 2004. p. 39-53.

VIEIRA, B. C.; RAMOS, H. Aplicação do modelo Shalstab para mapeamento da suscetibilidade a escorregamentos rasos em Caraguatatuba, Serra do Mar (SP). Revista do Departamento de Geografia, v. 29, p. 161-174. 2015.

VIEIRA, B. C. Previsão de escorregamentos translacionais rasos na Serra do Mar (SP) a partir de modelos matemáticos em bases fisicas. Tese (Doutorado em Geografia) - Instituto de Geociências, Universidade Federal do Rio de Janeiro, Rio de Janeiro, 2007.

ZAIDAN, R. T.; FERNANDES, N. F. Zoneamento de suscetibilidade a escorregamentos rasos em encostas aplicado à Bacia de Drenagem Urbana do córrego do Independência - Juiz de Fora (MG). Revista Brasileira de Geomorfologia. v. 10, n. 2, p. 57-76, 2009. 